\title{
Position zur Langzeit-Sauerstofftherapie
}

\section{Statement to Long Term Oxygen Therapy}

Autoren

Institute
H. Magnussen ${ }^{1}$, R. W. Hauck ${ }^{2}$, H. Worth ${ }^{3}$, F. J. F. Herth ${ }^{4}$

Die Institutsangaben sind am Ende des Beitrags gelistet.
Bibliografie

DOI http://dx.doi.org/

10.1055/s-0034-1377329

Online-Publikation: 21.7.2014

Pneumologie 2014; 68: 591-593

(c) Georg Thieme Verlag KG

Stuttgart · New York

ISSN 0934-8387

Korrespondenzadresse

Prof. Dr. med. H. Magnussen

Pneumologisches

Forschungsinstitut an der

LungenClinic Grosshansdorf

Wöhrendamm 80

22927 Großhansdorf

magnussen@pulmoresearch.de

\section{Einleitung \\ $\nabla$}

Die Erstfassung der Leitlinie zu Langzeit-Sauerstofftherapie wurde 2001 [1] entsprechend der methodischen Empfehlungen zur Bearbeitung von Leitlinien der Diagnostik und Therapie der Arbeitsgemeinschaft der wissenschaftlichen medizinischen Fachgesellschaft (AWMF) erstellt. Die Aktualisierung der Leitlinie erfolgte 2008 [2] auf der Grundlage einer Konsensuskonferenz 2007. Im Januar 2009 wurde eine Studie zur Langzeitsauerstofftherapie (Effectiveness of Long-term Oxygen Therapy in Treating People With Chronic Obstructive Pulmonary Disease, LOTT) mit insgesamt 737 Patienten in den Vereinigten Staaten begonnen [3]. Bis zum Dezember 2015 wird die Studie voraussichtlich abgeschlossen sein. $\mathrm{Zu}$ den Einschlusskriterien gehören eine COPD mit einer $\mathrm{FEV}_{1}<70 \%$ des Sollwertes und einer $\mathrm{O}_{2}$-Sättigung zwischen $89 \%$ und $93 \%$ in Ruhe oder einer Belastungshypoxie mit Abfall der $\mathrm{O}_{2}$-Sättigung unter 90\% über mehr als 10 Sekunden im 6-MinutenGehtest. Patienten werden dann entweder in einen Arm mit oder ohne Sauerstoffversorgung randomisiert. Im zweiten Arm der Studie werden die Patienten zunächst nicht mit Sauerstoff behandelt, sondern erhalten diese Therapie erst, wenn sie eine schwere Ruhehypoxämie entwickelt haben.

Die Patienten werden mindestens ein und höchstens sieben Jahre in der Studie betreut. Der primäre Studienendpunkt ist die Zeit bis zum Tod oder bis zu einer Krankenhauseinweisung mit einer Nachbeobachtung von bis zu sieben Jahren. Sekundäre Endpunkte sind Lebensqualität, krankheitsspezifische Lebensqualität, Überleben, Ernährungsstatus, Schlafqualität, 6-Minuten-Gehstrecke, Exazerbationen und Kosten (ClinicalTrials.gov Identifier: NCT00692198).
Diese Studie bildet einen Meilenstein in der Erforschung therapeutischer Methoden der Langzeitsauerstofftherapie und kann die Versorgungsempfehlungen bzgl. einer Langzeitsauerstofftherapie grundlegend verändern. Die Deutsche Gesellschaft für Pneumologie und Beatmungsmedizin hat sich daher entschlossen, die Bearbeitung der S2-Leitlinie zu diesem Thema bis zum Erscheinen dieser Daten zu prolongieren.

\section{Derzeitige Indikation zur Langzeit-Sauerstofftherapie $\nabla$}

Die nationalen Leitlinien unterscheiden sich nur unwesentlich von den internationalen Empfehlungen zur Langzeit-Sauerstofftherapie.

\section{COPD mit schwerer Hypoxämie}

Die derzeitige Indikation zur LTOT leitet sich aus zwei Studien ab, die in den frühen 1980er Jahren durchgeführt wurden. Diese bedeutsamen Studien sind als MRC (British Medical Research Council Long-Term Domiciliary Oxygen Therapy Trial) [5] und NOTT (Nocturnal Oxygen Therapy Trial) [4] bekannt geworden.

\section{MRC-Studie}

In der MRC-Studie wurden 87 Patienten mit chronischer Bronchitis und/oder Lungenemphysem untersucht (FEV-Wert zwischen 0,5 und 0,75 Liter). Der $\mathrm{PaO}_{2}$-Wert betrug 49,4 bis $51,8 \mathrm{mmHg}$ und der $\mathrm{PaCO}_{2}$-Wert 56 bis $60 \mathrm{mmHg}$. Die Patienten waren jünger als 70 Jahre und hatten ein Cor pulmonale. Die nasale Sauerstofftherapie erfolgte mit 21/min für mindestens 15 Stunden täglich. Die Patienten wurden über einen Zeitraum von 5 Jahren beobachtet. 19 der 42 sauerstoffbehandelten Patienten verstarben, im Vergleich zu 30 der 45 Kontrollpatienten, die keinen Sauerstoff 
erhielten und an denen Kontrollmessungen durchgeführt wurden. Die Mortalität wurde durch die LTOT günstig beeinflusst, allerdings fand sich kein statistisch signifikanter Unterschied in der Änderung von $\mathrm{FEV}_{1}, \mathrm{PaO}_{2}, \mathrm{PaCO}_{2}$, Hämatokrit oder pulmonal arteriellem Druck.

Die Chronic Obstructive Pulmonary Disease Mega-Analysis hat in der Ontario Health Technology Series 2012 die Frage untersucht, wie die Effektivität und Kosteneffizienz der LTOT im Vergleich zu COPD-Patienten ohne LTOT zu bewerten ist (www.hqontario.ca/ en/mas/tech/pdfs/2012/rev_LTOT_March.pdf). Bei COPD-Patienten mit schwerer Hypoxämie kam die Arbeitsgruppe zu dem Schluss, dass die LTOT, sofern sie mindestens ca. 15 Stunden pro Tag durchgeführt wird, die all-cause-mortality senkt. Die Autoren beziehen sich hier ausschließlich auf die MRC-Studie, da sie einen Kontrollarm enthält. Es wird weiterhin betont, dass die Senkung der all-cause-mortality vermutlich darauf zurückzuführen ist, dass bei diesen Patienten kardiovaskuläre Komorbiditäten vorgelegen haben. Der Effekt auf das Überleben war statistisch signifikant (12\% vs. 29\% jährliche Mortalität in der Kontrollgruppe; $\mathrm{p}=0,04)$.

\section{NOTT-Studie}

In der NOTT-Studie wurden 203 hypoxämische COPD-Patienten untersucht, die randomisiert entweder kontinuierlich Sauerstoff über 16 Stunden oder eine nächtliche Sauerstofftherapie erhielten. Die Patienten wurden über mindestens 12 Monate beobachtet, um den Effekt des Sauerstoffs auf das Überleben, den pulmonalen vaskulären Widerstand, die Lebensqualität und neuropsychologische Funktionen zu untersuchen. Die Mortalität lag nach 24 Monaten in der Patientengruppe mit nur nächtlicher Sauerstofftherapie mit 40,8\% signifikant höher im Vergleich zu $22,4 \%$ in der Gruppe, die kontinuierlich Sauerstoff $(17,7 \pm 4,8 \mathrm{~h} / \mathrm{d})$ erhielt $(p=0,01)$. Auch der Hämatokrit lag bei den Patienten unter kontinuierlicher Sauerstofftherapie hoch signifikant niedriger $(p=0,008)$ im Vergleich zur nächtlichen Therapie. Gleiches gilt auch für den pulmonal vaskulären Widerstand. Andere Parameter wie $\mathrm{FEV}_{1}$, pulmonal arterieller Druck, Lungenvolumina oder die arteriellen Blutgase waren nicht unterschiedlich in beiden Patientengruppen.

\section{Statement}

Aufgrund fehlender neuerer Daten bei Patienten mit schwerer Hypoxämie leitet sich die Indikation der LTOT bei COPDPatienten mit schwerer Hypoxämie unverändert positiv aus den Ergebnissen der NOTT- und MRC-Studie ab.

\section{COPD mit mäßiger bis moderater Hypoxämie}

Gorecka et al. [6] und Haidl et al. [7] untersuchten den Effekt der LTOT bei Patienten mit milder bis mäßiger Hypoxämie. Während Gorecka et al. eine Überlebensanalyse durchführten, untersuchten Haidl et al. den Einfluss der LTOT auf die körperliche Belastbarkeit und das Überleben. Die Gorecka-Studie belegt, dass die $\mathrm{PaO}_{2}$-Einschlusskriterien in der gültigen Leitlinie sinnvoll sind, da Patienten mit nur geringer bis mäßiger Hypoxämie (hier im Mittel $61 \mathrm{mmHg}$ ) nicht von dieser Therapieform profitieren. In der Studie von Haidl zeigte sich bei den 28 untersuchten Patienten $\left(\mathrm{PaO}_{2}\right.$ im Mittel $\left.67 \mathrm{mmHg}\right)$ eine deutliche Verbesserung sowohl der Dyspnoe als auch der Belastbarkeit im Endurance Test, allerdings ohne Einfluss auf das Überleben.

\section{Statement}

LTOT verbessert das Überleben bei COPD-Patienten mit schwerer Hypoxämie, während die Langzeitsauerstofftherapie keinen Einfluss auf das Überleben von Patienten mit mäßiger bis moderater Hypoxämie hat. Hinweise gibt es für eine Verbesserung der körperlichen Leistungsfähigkeit durch LTOT bei dieser Patientengruppe.

Sauerstofftherapie bei COPD ohne Ruhe-, aber mit belastungsinduzierter Hypoxämie

Drummond et al. [8] untersuchten in einer retrospektiven Analyse bei 1215 Patienten mit COPD, die am National Emphysema Treatment Trial teilgenommen hatten, den Effekt der LTOT trotz fehlender Ruhe-Hypoxämie. 160 normoxämische COPD-Patienten nutzten eine kontinuierliche Sauerstofftherapie und wurden mit 266 normoxämischen COPD-Patienten verglichen, die keinen Sauerstoff anwendeten. Die Patienten, die Sauerstoff benutzten, wiesen mehr Luftnot, eine schlechtere Lebensqualität, häufigere Sauerstoffentsättigungen während körperlicher Belastung und eine erhöhte Todesrate auf. In der Datenanalyse zeigte sich, dass diese Patienten eine schlechtere Lungenfunktion und einen pathologischen BMI hatten, welche zu einer erhöhten Mortalität führten. Die Nutzung von Sauerstoff wurde als Surrogatmarker für ein fortgeschritteneres Krankheitsstadium und eine höhere Mortalität gewertet. Obwohl sich die Mortalität unter $\mathrm{O}_{2}$-Gabe nicht veränderte, verbesserte sich die Lebensqualität (SGRQ: $\mathrm{p}<0,0001)$.

Moore et al. [9] konnten bei 143 Patienten keinen Effekt der Sauerstofftherapie bei körperlicher Belastung auf Luftnot, gesundheitsbezogene Lebensqualität, Depressionsskalen sowie funktionelle Daten nachweisen. Bei diesen Ergebnissen ist jedoch zu berücksichtigen, dass nur ein Drittel der Probanden eine erhebliche Entsättigung unter Belastung aufwies $\left(\mathrm{SaO}_{2}<88 \%\right)$. Die Autoren verwendeten Sauerstoffzylinder mit einem Gewicht von $4,2 \mathrm{~kg}$. Es ist denkbar, dass der Einsatz moderner, portabler Sauerstoffsysteme vermutlich zu besseren Ergebnissen geführt hätte.

In den Leitlinien zur Langzeit-Sauerstofftherapie der Deutschen Gesellschaft für Pneumologie und Beatmungsmedizin wird empfohlen, bei Patienten, die nur während Belastung eine Hypoxämie aufweisen und bei denen eine Sauerstoffapplikation zu einem Anstieg des $\mathrm{PaO}_{2}$-Wertes $\geq 60 \mathrm{mmHg}$ und $\mathrm{zu}$ einer Verbesserung der Belastbarkeit führen, eine Sauerstofftherapie mit mobilen Geräten durchzuführen. Dieses Vorgehen entspricht auch den Empfehlungen von Koehler et al. [10].

Obwohl internationale Leitlinien zur Langzeit-Sauerstofftherapie überwiegend analoge Empfehlungen aussprechen, ist die Sauerstofftherapie bei Patienten umstritten, die in Ruhe normoxämisch sind und nur während körperlicher Belastung oder Aktivitäten des täglichen Lebens entsättigen [11].

\section{Statement}

Die Studienlage ist nicht eindeutig. Es zeigt sich jedoch, dass bei Patienten, die in Ruhe normoxämisch sind, aber eine Belastungshypoxämie entwickeln, die LTOT eine Verbesserung der Symptomatik und damit auch der Lebensqualität und der Belastbarkeit bewirkt. 


\section{COPD und Komorbidität}

Es ist bekannt, dass Patienten mit COPD Komorbiditäten aufweisen, die auch ohne COPD mit Luftnot bei körperlicher Belastung einhergehen können. Der Einfluss kardialer Komorbidität wurde bereits dargestellt. Weitere Untersuchungen, die einen Effekt der Sauerstofftherapie bei körperlicher Belastungen bei COPD-Patienten mit und ohne Komorbiditäten untersuchen, stehen aus.

\section{LTOT und Dyspnoe in palliativer Situation}

Hierzu gibt es nur wenige randomisierte Studien, die sich mit der Reduktion der Luftnot als Outcome-Parameter bei Patienten in palliativer Situation beschäftigen $[12,13]$. Kürzlich wurde gezeigt, dass die Sauerstofftherapie bei Patienten im Endstadium einer Tumorerkrankung nur einen geringen Einfluss auf die Verminderung der Luftnot zeigte $[13,14]$. In der Palliativmedizin sollte daher bei jedem Einzelfall sorgfältig geprüft werden, ob eine LTOT tatsächlich sinnvoll ist.

\section{Sauerstofflangzeittherapie bei Lungengerüst- erkrankungen}

Hypoxämie ist häufig bei Patienten mit Lungengerüsterkrankungen, insbesondere bei der idiopathischen Lungenfibrose. Der Abfall des Sauerstoffpartialdruckes (Sauerstoffsättigung) ist bei diesen Patienten besonders ausgeprägt und gilt als ein prognostischer Marker der Erkrankung [15,16].

Es ist bisher nicht nachgewiesen worden, ob die Langzeit-Sauerstofftherapie bei Patienten mit Lungengerüsterkrankungen das Überleben günstig beeinflusst. Die Anwendung der LangzeitSauerstofftherapie bei Patienten mit Lungengerüsterkrankungen wird mit den Erfahrungen begründet, die bei Patienten mit COPD und Lungenemphysem gewonnen wurden. Die Gabe von Sauerstoff bei körperlicher Belastung ist bei diesen Patienten hilfreich.

\section{Statement}

Die Untersuchungen, die zur LTOT bei Patienten mit Lungenfibrose vorliegen, zeigen, dass die Sauerstoffgabe zur Verminderung der Belastungshypoxämie sinnvoll ist.

\section{Offene Fragen}

Die Leitlinien zur Langzeit-Sauerstofftherapie der Deutschen Gesellschaft für Pneumologie und Beatmungsmedizin spiegeln das gegenwärtige Wissen über die Langzeit-Sauerstofftherapie wider. Bereits 2007 wurden die zukünftigen Forschungsfragen um die Langzeit-Sauerstofftherapie bei COPD innerhalb eines Workshops des National Heart, Lung, and Blood Institute diskutiert. Leider sind die meisten dieser Fragen auch heute noch unbeantwortet, da seit 1981 der Einfluss der LTOT auf die Mortalität nicht erneut untersucht worden ist.

Die medizintechnische Industrie hat in den letzten Jahren erhebliche Fortschritte in der Entwicklung von Sauerstoffsystemen gemacht. Die Langzeit-Sauerstofftherapie kann jetzt sowohl mit stationären als auch mobilen Sauerstoffkonzentratoren und stationär und mobil anwendbarem Flüssigsauerstoff durchgeführt werden. Koehler und Mitarbeiter [10] haben kürzlich die Langzeitsauerstofftherapie unter dem Gesichtspunkt dargestellt, was Arzt, Versorger und Krankenkassen bei der Anwendung zu berücksichtigen haben. Diese Darstellung ist daher eine wichtige Ergänzung zum alltäglichen Umgang mit der LTOT.
Die Ergebnisse der Effectiveness of Long-term Oxygen Therapy in Treating People With Chronic Obstructive Pulmonary Disease (The Long-term Oxygen Treatment Trial) müssen nun abgewartet werden, bevor eine Überarbeitung der Leitlinie sinnvoll erscheint.

\section{Interessenkonflikt}

\section{$\nabla$}

Die Autoren geben an, dass kein Interessenkonflikt besteht.

\section{Institute}

${ }_{1}^{1}$ Pneumologisches Forschungsinstitut an der LungenClinic Grosshansdorf ${ }^{2}$ LungenZentrum SüdOst, Kreisklinik Bad Reichenhall

${ }^{3}$ Klinikum Fürth, Medizinische Klinik I

${ }^{4}$ Thoraxklinik und Translational Lung Research Center Heidelberg, Mitglied des Deutschen Zentrums für Lungenforschung, Universitätsklinik Heidelberg

\section{Literatur}

1 Magnussen H, Goeckenjan G, Koehler D et al. Leitlinien zur LangzeitSauerstofftherapie. Pneumologie 2001; 55: 454-464

2 Magnussen H, Kirsten AM, Koehler D et al. Leitlinien zur Langzeit-Sauerstofftherapie - Deutsche Gesellschaft für Pneumologie und Beatmungsmedizin e.V. Pneumologie 2008; 62: 748 - 756

3 Stoller JK, Panos RJ, Krachman S et al. Oxygen therapy for patients with COPD: current evidence and the long-term oxygen treatment trial. Chest 2010; 138: 179-187

4 Nocturnal Oxygen Therapy Trial Group. Continuous or nocturnal oxygen therapy in hypoxemic chronic obstructive lung disease: a clinical trial. Nocturnal Oxygen Therapy Trial Group. Ann Intern Med 1980; 93: $391-398$

5 Medical Research Council Working Party. Long term domiciliary oxygen therapy in chronic hypoxic cor pulmonale complicating chronic bronchitis and emphysema. Report of the Medical Research Council Working Party. Lancet 1981; 1: 681 -686

6 Gorecka D, Gorzelak K, Sliwinski P et al. Effect of long-term oxygen therapy on survival in patients with chronic obstructive pulmonary disease with moderate hypoxaemia. Thorax 1997; 52: 674-679

7 Haidl P, Clement P, Wiese $C$ et al. Long-term oxygen therapy stops the natural decline of endurance in COPD patients with reversible hypercapnia. Respiration 2004; 71: 342 - 347

8 Drummond MB, Blackford AL, Benditt OJ et al. Continuous oxygen use in nonhypoxemic emphysema patients identifies a high-risk subset of patients: retrospective analysis of the National Emphysema Treatment Trial. Chest 2008; 134: 497-506

9 Moore RP, Berlowitz DJ, Denehy L et al. A randomised trial of domiciliary, ambulatory oxygen in patients with COPD and dyspnoea but without resting hypoxaemia. Thorax 2011; 66: $32-37$

10 Koehler $U$, Hildebrandt 0 , Jerrentrup $L$ et al. Die Langzeit-SauerstoffTherapie (LTOT) - Was sollten Arzt, Versorger und Krankenkasse wissen? Pneumologie 2014; 68: 193-198

11 Criner GJ. Ambulatory home oxygen: what is the evidence for benefit, and who does it help? Respir Care 2013; 58: 48 -64

12 Booth S, Kelly MJ, Cox NJ et al. Does oxygen help dyspnea in patients with cancer? Am J Respir Crit Care Med 1996; 153: 1515-1518

13 Booth SR, Johnson M, Kite $S$ et al. The use of oxygen in the palliation of breathlessness. A report of the expert working group of the Scientific Committee of the Association of Palliative Medicine. Respir Med 2004 98: $66-77$

14 Nava S, Ferrer M, Esquinas A et al. Palliative use of non-invasive ventilation in end-of-life patients with solid tumours: a randomised feasibility trial. Lancet Oncol 2013; 14: 219-227

15 Flaherty KR, Andrei AC, Murray $S$ et al. Idiopathic pulmonary fibrosis: prognostic value of changes in physiology and six-minute-walk test. Am J Respir Crit Care Med 2006; 74: 803-809

16 Hallstrand TS, Boitano LJ, Johnson WC et al. The timed walk test as a measure of severity and survival in idiopathic pulmonary fibrosis. Eur Respir J 2005; 25: 96-103 